\title{
Physical and Sexual Intimate Partner Violence Among Women in Methadone Maintenance Treatment
}

Nabila El-Bassel [a], Louisa Gilbert [a], Victoria Frye [a], Elwin Wu [a], Hyun Go [a], Jennifer Hill [a], Beverly L. Richman [b]

[a] Social Intervention Group, Columbia University School of Social Work

[b] Beth Israel Medical Center, New York, NY

This research was supported by Grant DA11027 from the National Institute on Drug Abuse. We acknowledge the contributions of the staff of the Methadone Maintenance Treatment Program at the Beth Israel Medical Center, New York, who made this work possible. The content presented in this article is solely the responsibility of the authors and does not necessarily represent the official views of the National Institute on Drug Abuse, the Beth Israel Medical Center, or the Columbia University School of Social Work.

Correspondence concerning this article should be addressed to Nabila El-Bassel, Columbia University School of Social Work, 622 West 113th Street, New York, NY 10025. Email: ne5@columbia.edu.

\begin{abstract}
Although several studies have examined the relationship between intimate partner violence (IPV) and drug use among women in drug treatment programs, more information is needed to delineate differences, as a function of the specific drug used. Data from a random sample of 416 women attending methadone programs were analyzed to elucidate the differential associations between IPV and use of the following: marijuana only, cocaine only, heroin only, or cocaine and heroin. Prevalence of IPV among this sample far exceeded estimates from the general population. After adjusting for sociodemographic variables, use of cocaine only was significantly associated with an increased likelihood of experiencing IPV compared with no drug use. Similar results were found for women using both cocaine and heroin.
\end{abstract}

\section{Introduction}

Recognition of intimate partner violence (IPV) as a significant public health problem for women in drug treatment has emerged over the last decade. Past-year prevalence rates of IPV among women in drug treatment have been found to range between $25 \%$ and $57 \%$ (Brewer, Fleming, Haggerty, \& Catalano, 1998; Chermack, Fuller, \& Blow, 2000; El-Bassel, Gilbert, Wada, Witte, $\&$ Schilling, 2000). These rates of received IPV are substantially higher than the range of $1.5 \%$ to $16 \%$ prevalence rates found in epidemiological surveys of community-based samples (Caetano, Nelson, \& Cunradi, 2001; Tjaden \& Thoennes, 1998). Furthermore, a growing body of evidence suggests that interpersonal stress and relationship conflict are major triggers for relapse among women in drug treatment (Leshner, 1998; McKay, Rutherford, Cacciola, KabasakalianMcKay, \& Alterman, 1996) and that IPV may result in continued drug use, relapse, and attrition 
from drug treatment among women (Gilbert, El-Bassel, Rajah, Foleno, \& Frye, 2001; Gil-Rivas, Fiorentine, Anglin, \& Taylor, 1997; Melchior, Huba, Brown, \& Slaughter, 1999).

This study addresses some of the critical gaps in knowledge on the prevalence of IPV and on the relationship between drug use and IPV among women in drug treatment. In this study we (a) describe the prevalence rates of different types of IPV (i.e., physical, sexual, and injurious) in the past 6 months and lifetime; (b) describe univariate associations between sociodemographic and drug use factors and experience of IPV; (c) examine the differential associations between women's drug use and IPV, depending on type of drug(s) women used-marijuana only, crack cocaine only, heroin only, or both crack cocaine and heroin - and their experience of different types and severity of IPV, while adjusting for potentially confounding sociodemographic characteristics.

\section{Method}

Random Sampling and Recruitment Procedures

We randomly selected 753 women from the total population of women who were enrolled in 14 methadone maintenance treatment clinics in New York City. Eligibility criteria for this study were being female and between the ages of 18 and 55; being in methadone maintenance treatment for at least 3 months; and during the past year, having had a sexual or dating relationship with someone whom the woman described as her boyfriend, girlfriend, spouse, regular sexual partner, or the father of her children.

A total of 559 women agreed to participate in a 15-min screening interview, and 427 met eligibility criteria. Of those eligible, 416 (97\%) women agreed to participate. All participants signed a consent form that detailed confidentiality procedures and their rights as research participants. The Institutional Review Boards of the participating methadone maintenance treatment programs (MMTPs) and Columbia University (the research institution) approved the protocol for this study. Participants were compensated $\$ 5$ for completing the screening interview and $\$ 20$ for completing the baseline interview.

\section{Measurement}

Trained interviewers conducted face-to-face structured interviews with participants. The baseline questionnaire assessed socio-demographic information, including age, ethnicity, level of education, marital status, having more than one partner, and current employment status.

We assessed drug use with the Drug Use and Risk Behavior Questionnaire (El-Bassel, Ivanoff, Schilling, Gilbert, \& Chen, 1995). This questionnaire assesses frequency of heroin, crack, cocaine, and marijuana use in the prior 6 months with a 6-point Likert scale.

We also inquired about IPV experienced in the prior 6 months as well as lifetime, using the Minor and Severe Physical Assault, Sexual Coercion, and Injury Scales of the Revised Conflict Tactics Scales (Straus, Hamby, Boney-McCoy, \& Sugarman, 1996).

Data Analysis 
We calculated the prevalence of IPV by the types listed in Table 1 .

Table 1

Prevalence of Intimate Partner Violence (IPV) by Type,

Severity, and Time Period

\begin{tabular}{|c|c|c|c|c|}
\hline \multirow[b]{2}{*}{ Type of IPV } & \multicolumn{2}{|c|}{ Past 6 months } & \multicolumn{2}{|c|}{ Lifetime } \\
\hline & $n$ & $\%$ & $n$ & $\%$ \\
\hline \multicolumn{5}{|l|}{ Sexual } \\
\hline Minor & 123 & 29.7 & 185 & 44.7 \\
\hline Severe & 19 & 4.6 & 54 & 13.0 \\
\hline All (minor or severe) & 127 & 30.7 & 192 & 46.4 \\
\hline \multicolumn{5}{|l|}{ Physical } \\
\hline Minor & 111 & 26.8 & 211 & 51.0 \\
\hline Severe & 58 & 14.0 & 155 & 37.4 \\
\hline All (minor or severe) & 128 & 30.9 & 218 & 52.7 \\
\hline \multicolumn{5}{|l|}{ Injurious } \\
\hline Minor & 72 & 17.4 & 159 & 38.4 \\
\hline Severe & 33 & 8.0 & 92 & 22.2 \\
\hline All (minor or severe) & 76 & 18.4 & 173 & 41.8 \\
\hline \multicolumn{5}{|c|}{ Any (sexual and physical or injurious) } \\
\hline Minor & 184 & 44.4 & 356 & 86.1 \\
\hline Severe & 77 & 18.6 & 301 & 72.7 \\
\hline All (minor or severe) & 193 & 46.6 & 363 & 87.6 \\
\hline
\end{tabular}

Note. $\quad N=414$.

Women who reported experiencing one or more abusive acts by an intimate partner on the Minor and Severe Physical Assault, Injury, or Sexual Coercion subscales of the Revised Conflicts Tactics Scale in the past 6 months were considered "recently abused" $(\mathrm{n}=193)$. Women who indicated that they had experienced IPV on these subscales in the past, but not in the past 6 months, and women who never experienced IPV, were considered "non-recently abused" ( $n=$ 223).

We compared recently abused versus non-recently abused women, using univariate logistic regression analysis to estimate unadjusted odds ratios (ORs) and 95\% confidence intervals (CIs) for the sociodemographic and drug use factors associated with recent IPV.

We used logistic regression analyses to examine whether women's use of marijuana, crack cocaine, and/or heroin in the past 6 months was associated with different types of IPV after adjusting for potentially confounding sociodemographic variables (i.e., age, education, marital status, ethnicity, and having more than one intimate partner in the past year), which were selected on the basis of whether previous research indicated a relationship with IPV (Caetano et al., 2001; Chermack, Walton, Fuller, \& Blow, 2001).

In this logistic regression model, sociodemographic background variables are entered with four mutually exclusive drug use type categories for women: (a) marijuana only - marijuana use in the past 6 months, but not crack cocaine or heroin; (b) crack cocaine only-crack cocaine use in the past 6 months, but not heroin; (c) heroin only-heroin use in the past 6 months, but not crack cocaine; and (d) crack cocaine and heroin - heroin and crack cocaine use in the past 6 months. Participants who used marijuana in combination with crack cocaine or heroin in the past 6 
months were categorized according to their use of crack cocaine and heroin. The reference group for the drug use variable is women who reported no use of marijuana, crack cocaine, or heroin in the past 6 months. Our rationale for the use of these specific drugs is based on previous research that found high rates of crack cocaine use in combination with heroin use, as well as significant rates of marijuana use among women in MMTPs (Brewer et al., 1998; El-Bassel et al., 1998). These mutually exclusive drug use categories may accommodate for the identification of differential associations between IPV and drug use based on the specific drug used by women.

Two women did not provide self-reported IPV data. For regression analyses, we used multiple imputation to deal with missing data. We performed the multiple imputation using Amelia for Windows (King, Honaker, Joseph, \& Scheve, 2000).

\section{Results}

Prevalence of IPV

Prevalence of different types of IPV in the past 6 months and lifetime are reported in Table 1. Among the 414 women who provided self-reported IPV data, $88 \%$ reported sustaining an injury or experiencing at least one incident of physical or sexual IPV in their lifetime. Almost three quarters $(73 \%)$ of the women indicated that they had experienced severe injurious, physical, or sexual IPV in their lifetime. About one half (47\%) of the women reported injurious, physical, or sexual IPV in the past 6 months, and about one-fifth (19\%) indicated that they had experienced severe physical or sexual IPV or had sustained a severe injury by an intimate partner in the past 6 months. The prevalence estimates of received IPV broken down by type, severity, and time frame are provided in Table 1.

\section{Sociodemographic Characteristics}

The mean age of the women was 39.9 years ( $\mathrm{SD}=6.7$ years), $20 \%$ were married, and $21 \%$ had had more than one sexual partner in the past year. The majority were Latina $(48 \%, \mathrm{n}=198)$ or African American $(32 \%, \mathrm{n}=128)$. More than half $(58 \%, \mathrm{n}=240)$ had completed high school.

Bivariate logistic regression analyses of the data indicated that any physical, sexual, or injuryrelated IPV in the past 6 months was associated with being a high school graduate $(\mathrm{OR}=1.7$, 95\% CI: 1.0, 2.8), never married (OR $=1.7,95 \% \mathrm{CI}: 1.0,3.0)$ and having more than one intimate partner in the past year $(\mathrm{OR}=3.0,95 \% \mathrm{CI}: 1.8,5.0)$. Recently abused women were less likely than their counterparts to be White $(\mathrm{OR}=0.58,95 \% \mathrm{CI}=0.35,0.98)$.

Current Drug Use

Thirty-eight percent of the women reported using heroin in the past 6 months, $40 \%$ reported using crack cocaine, and one quarter (25\%) reported using marijuana. One quarter $(25 \%)$ reported using both crack cocaine and heroin.

Relationship Between Type of Drug Used and Type of IPV

Results from analyses regarding the relationships between women's drug use and experiencing various types of IPV in the prior 6 months are presented in Table 2. 
Table 2

Adjusted Odds Ratios (AORs) of Different Types of Drug Use and Experience of Intimate Partner Violence (IPV) in the Past 6 Months

\begin{tabular}{|c|c|c|c|c|c|c|c|c|}
\hline \multirow[b]{2}{*}{ Drug use ${ }^{a}$} & \multicolumn{2}{|c|}{ Any IPV } & \multicolumn{2}{|c|}{ Any sexual IPV } & \multicolumn{2}{|c|}{ Any physical IPV } & \multicolumn{2}{|c|}{ Any severe IPV } \\
\hline & $\mathrm{AOR}^{\mathrm{b}}$ & (CI) & $\mathrm{AOR}^{\mathrm{b}}$ & (CI) & $\mathrm{AOR}^{\mathrm{b}}$ & (CI) & $\mathrm{AOR}^{\mathrm{b}}$ & (CI) \\
\hline Marijuana only & 1.4 & $(0.6,3.8)$ & 1.1 & $(0.4,2.8)$ & 1.3 & $(0.5,3.3)$ & 1.6 & $(0.5,4.7)$ \\
\hline Crack cocaine only & 2.3 & $(1.2,2.6)$ & 2.2 & $(1.2,4.3)$ & 2.3 & $(1.2,4.4)$ & 2.0 & $(0.9,4.6)$ \\
\hline Heroin only & 0.9 & $(0.5,2.7)$ & 0.8 & $(0.4,1.6)$ & 1.0 & $(0.5,2.0)$ & 1.2 & $(0.5,2.9)$ \\
\hline Crack cocaine \& heroin & 2.2 & $(1.3,2.3)$ & 2.3 & $(1.3,4.0)$ & 2.3 & $(1.3,4.0)$ & 3.1 & $(1.6,6.1)$ \\
\hline
\end{tabular}

Note. $\quad N=416 . \mathrm{CI}=95 \%$ confidence interval.

a The reference group for all four mutually exclusive women's drug use categories are women who reported no use of marijuana, crack cocaine, or heroin in the past 6 months. ' Adjusted for women's age, ethnicity, education, marital status, and having more than one partner.

This table presents the odds ratios for various types of IPV by four mutually exclusive recent drug use categories for the women-(a) marijuana only, (b) crack cocaine only, (c) heroin only, and (d) crack cocaine and heroin - adjusting for age, ethnicity, marital status, education, and having more than one intimate partner. Compared to women who reported no use of illicit drugs, women who reported only using crack cocaine were more likely to report experiencing any IPV, sexual IPV, and physical IPV in the past 6 months. Women who reported crack cocaine and heroin use were also more likely than women who did not use illicit drugs to report experiencing any IPV, sexual IPV, physical IPV, and injurious IPV in the past 6 months. No significant differences were observed between women's use of only marijuana or only heroin compared to no use of illicit drugs for any of the different types of IPV in the past 6 months.

\section{Discussion}

To our knowledge, this is the first study to examine the prevalence of different types of IPV and the associations between various types of drug use using a random sample of low-income, urban women attending MMTPs. The past 6-month prevalence rates of physical, injurious, or sexual IPV among this random sample was as high or higher than the range of past-year prevalence rates of IPV found in previous studies of nonrandom samples of women in drug treatment. The significant associations between crack cocaine use alone or in conjunction with heroin and all types of IPV suggest that multiple mechanisms may link crack cocaine use and IPV, as indicated by the previous research mentioned earlier. The lack of any observed significant associations between only using heroin and IPV is also consistent with previous studies (Brewer et al., 1998; El-Bassel et al., 2000). Contrary to previous research (Brewer et al., 1998; El-Bassel et al., 2000), this study did not find any significant associations between marijuana use and different types of IPV. The lack of association found in this study may be a result of not including polydrug marijuana users with marijuana-only users.

The causal relationship between IPV and illicit drug use remains unclear in this cross-sectional study. Longitudinal studies among women in methadone maintenance treatment, as well as in other drug abuse treatment programs, are needed to test causal hypotheses more definitively, that is, whether IPV leads to subsequent crack cocaine use, crack cocaine use leads to subsequent 
IPV, or whether a third factor (e.g., exchange of sex for money or drugs) drives both crack cocaine use and IPV.

Although the current study used data from a random sample of women in MMTPS that is larger than previous studies, which also typically used nonrandom samples, the findings may not be generalizable to other populations of women in drug treatment. In addition, further analyses are needed to account better for the frequency of drug use, because associations between drug use patterns and IPV may change on the basis of the extent of abuse or addiction to illicit drugs. Failure to control for the drug use of the women's partners is another limitation of this study.

Despite these limitations, this study has implications for addressing the co-occurring problems of substance abuse and IPV among low-income, urban women in MMTPs. The high prevalence of recent and severe IPV among this random sample of women underscores the need to implement routine screening for IPV in MMTPs. Failure to identify and prevent IPV among women in drug treatment may jeopardize their safety as well as their recovery.

The different associations with IPV by type of drug use found in this study further suggest the importance of using a drug-specific approach, rather than a generic approach, to address comorbid substance abuse and IPV. Given the limitations on resources and time that often exist within methadone treatment programs, enhanced assessment and referral could be targeted toward women at higher risk, that is, female patients who need services for both opioid and crack cocaine abuse. In addition, standard protocols for assessment, safety planning, treatment, and referral to address the co-occurring problem of IPV with different types of drug use, particularly crack cocaine use, need to be designed, tested, and implemented.

\section{References}

Brewer, D. D., Fleming, C. B., Haggerty, K. P., \& Catalano, R. F. (1998). Drug use predictors of partner violence in opiate dependent women. Violence and Victims, 13, 107-115.

Caetano, R., Nelson, S., \& Cunradi, C. (2001). Intimate partner violence, dependence symptoms and social consequences from drinking among White, Black and Hispanic couples in the United States. American Journal on Addictions, 10 (Suppl.), 60-69.

Chermack, S. T., Fuller, B. E., \& Blow, F. C. (2000). Predictors of expressed partner and nonpartner violence among patients in substance abuse treatment. Drug and Alcohol Dependence, $58,43-54$.

Chermack, S. T., Walton, M. A., Fuller, B. E., \& Blow, F. C. (2001). Correlates of expressed and received violence across relationship types among men and women substance abusers. Psychology of Addictive Behaviors, 15, 140-151.

El-Bassel, N., Gilbert, L., Krishnan, S., Schilling, R. F., Gaeta, T., Purpura, S., \& Witte, S. S. (1998). Partner violence and sexual HIV-risk behaviors among women in an inner-city emergency department. Violence and Victims, 13, 377-393. 
El-Bassel, N., Gilbert, L., Wada, T., Witte, S. S., \& Schilling, R. F. (2000). Drug abuse and partner violence among women in methadone treatment. Journal of Family Violence, 15, 209225.

El-Bassel, N., Ivanoff, A., Schilling, R. F., Gilbert, L., \& Chen, D. R. (1995). Correlates of problem drinking among drug using incarcerated women. Addictive Behaviors, 20, 359-369.

Gilbert, L., El-Bassel, N., Rajah, V., Foleno, A., \& Frye, V. (2001). Linking drug related activities with experiences of partner violence: A focus group study of women in methadone treatment. Violence and Victims, 16, 517-536.

Gil-Rivas, V., Fiorentine, R., Anglin, M. D., \& Taylor, E. (1997). Sexual and physical abuse: Do they compromise drug treatment outcomes? Journal of Substance Abuse Treatment, 14, 351358.

King, G., Honaker, J., Joseph, A., \& Scheve, K. (2000). Analyzing incomplete political science data: An alternative algorithm for multiple imputation. American Political Science Review, 95, 49-69.

Leshner, A. (1998). Gender matters in drug abuse research. NIDA Notes, 13(4), 11.

McKay, J. R., Rutherford, M. J., Cacciola, J. S., Kabasakalian-McKay, R., \& Alterman, A. I. (1996). Gender differences in the relapse experiences of cocaine patients. Journal of Nervous and Mental Disease, 184, 616-622.

Melchior, L. A., Huba, G. J., Brown, V. B., \& Slaughter, R. (1999). Evaluation of the effects of outreach to women with multiple vulnerabilities on entry into substance abuse treatment. Evaluation and Program Planning, 22, 269-277.

Straus, M. A., Hamby, S. L., Boney-McCoy, S., \& Sugarman, D. B. (1996). The Revised Conflict Tactics Scales (CTS2): Development and preliminary psychometric data. Journal of Family Issues, 17, 283-316.

Tjaden, P., \& Thoennes, N. (1998). Prevalence, incidence, and consequences of violence against women: Findings from the National Survey of Violence Against Women Survey. (Report No. NCJ 172837). Washington, DC: U.S. Department of Justice. 